\title{
On solutions of the nonlinear heat equation using modified Lie symmetries and differentiable topological manifolds
}

\author{
JACOB MANALE \\ Department of Mathematical Sciences \\ University of South Africa \\ Corner Christiaan de Wet Street and Pioneer Avenue, 1709 Florida, Johannesburg, Gauteng Province \\ REPUBLIC OF SOUTH AFRICA
}

\begin{abstract}
In this paper, we present three simple analytical techniques for obtaining solutions of the nonlinear heat equations. The heat equations, both linear and nonlinear, are very important to the mathematical sciences. This is because they are reduced forms of many models, hard to solve directly. The techniques are based on Lie' symmetry group theoretical methods. The first is the pure Lie approach, followed by our modified Lie approach. The third is our differentiable topological manifolds approach. As an application, we determine the separation distance, in the quantum superposition principle, relevant to nanoscience.
\end{abstract}

Key-Words: - Nonlinear heat equation. Differential Equations. Lie Symmetry Analysis.

Received: February 5, 2021. Revised: September 13, 2021. Accepted: September 21, 2021. Published: September 30, 2021.

\section{Introduction}

The nonlinear heat equation

$$
w_{x x}-w_{t}=w_{x}^{2}
$$

can be reduced to the linear heat equation

$$
u_{x x}-u_{t}=0
$$

using the invertible transformation

$$
u=e^{\omega}-1
$$

the invertible transformation being

$$
\omega=\ln |u+1|
$$

The well-known and generally accepted solution for (2) is

$$
u=\left(C_{1} \frac{x}{t}+C_{2}\right) \frac{e^{\frac{x^{2}}{4 t}}}{\sqrt{t}}
$$

where $C_{1}$ and $C_{2}$ constants of integration. This solution happens to be impractical in many models. Our objective in this contribution is to demonstrate that there are other solutions, transferable to the non-linear case.
Historically, the Norwegian mathematician, Sophus Lie, is the first to develop Lie symmetry group theoretical methods, about three centuries ago, presented through his now famous paper [1]. Ovsiannikov [2], later revitalized it, in the $50 \mathrm{~s}$, The theory has since grown in leaps and bounds, advanced further by the likes of Ibragimov et. al., Leach et. al [3], Mahomed et. al. [4], Wafo Sof [5], Kara [6], Momoniat [7], Ibragimov et. al. [8], Gazizov et. al. [9], Bytev [10], Govinder and Edelstein [11]. The list is long. The procedure is simple. First, it is the determination of symmetries, followed by solutions. Ovsiannikov proposed group invariant solutions.

Our discomfort, which is the primary drive that led to us to develop our two techniques, is that these traditional methods, most often do not really solve the equations they should solve. Integrals are unresolved.

We demonstrate here how to avoid these problems. Our first technique dealing with solutions, involve mean value theorems, which comes naturally with the modified symmetries. The second is through differentiable topological manifolds, both used here, in this contribution.

We present a brief Lie symmetry analysis of (2) in Section 2. This is as a precursor for modified Lie 
symmetry analysis. The latter depends on some aspects the former.

In Section 3 we determine the modified symmetries for (2), based on the monomials established in the previous section. We include the definition and theorems necessary for generating these type of symmetries.

Section 4 is on the group invariant solutions, which determine using two procedures. First, we use mean value theorem. Next, we develop the same solutions through differentiable topological manifolds.

\section{A Lie symmetry analyses of (2)}

Lie symmetries result from point transformations. There are many transformations. We limit our attention to one-parameter point transformations. We begin with a definition.

Definition 1. Suppose $\left(x^{1} ; \cdots ; x^{N}\right)$ is a point in $\mathrm{IR}^{N}$, and is related to another $\left(\bar{x}^{1} ; \cdots ; \bar{x}^{N}\right)$ in $\overline{\mathrm{IR}}^{N}$ by

$\bar{x}^{i}=f\left(x^{1} ; \cdots ; x^{N} ; a\right), i=1, \cdots, N$,

an invertible transformation, such that $a$ is an infinitely small parameter. The transformation is called local one parameter point transformation.

A symmetry analysis of (2), then involves seeking one parameter local point transformations of the form

$$
\bar{t}=f(t, x, u, a), \bar{x}=g(t, x, u, a), \quad \bar{u}=
$$

$h(t, x, u, a)$,

where $a$ the parameter. An infinitesimally small parameter, as declared in the definition. The quantity $u$ is a function of the independent variables $t$ and $x$. The functions $f, g$ and $h$ represent the transformed variables $\bar{t}, \bar{x}$ and $\bar{u}$. The set of all such transformations forms a group, subject to

$$
\bar{t} \approx t+\xi^{1} a, \bar{x} \approx x+\xi^{2} a, \bar{u} \approx u+\eta a,
$$

where $\xi^{1}, \xi^{2}$ and $\eta$ are the infinitesimals. Introducing a symbol of infinitesimal transformations, we have the operator

$$
\begin{aligned}
& X=\xi^{1}(t, x, u) \frac{\partial}{\partial t}+\xi^{2}(t, x, u) \frac{\partial}{\partial x} \\
& \eta(t, x, u, a) \frac{\partial}{\partial u} .
\end{aligned}
$$

This corresponds to the Lie equations $\eta(t, x, u)$

$$
\frac{d \bar{t}}{d a}=\xi^{1}(t, x, u), \frac{d \bar{x}}{d a}=\xi^{2}(t, x, u), \frac{d \bar{u}}{d a}=
$$

with the initial conditions

$$
\left.\frac{d \bar{t}}{d a}\right|_{a=0}=t,\left.\quad \frac{d \bar{x}}{d a}\right|_{a=0}=x,\left.\quad \frac{d \bar{u}}{d a}\right|_{a=0}=u .
$$

Equation (2) admits a second-order generator

$$
X^{[2]}=X+\zeta_{t}^{1} \frac{\partial}{\partial u_{t}}+\zeta_{x x}^{2} \frac{\partial}{\partial u_{x x}},
$$

where the prolongations $\zeta_{t}^{1}$ and $\zeta_{u_{x x}}^{2}$ are given by

$$
\begin{aligned}
& \zeta_{t}^{1}=\eta_{t}+\left[\eta_{u}-\xi^{1}{ }_{t}\right] u_{t}-\xi^{2}{ }_{t} u_{x}- \\
& \xi^{1}{ }_{u}\left(u_{t}\right)^{2}-\xi^{2}{ }_{u} u_{t} u_{x},
\end{aligned}
$$

and

$$
\begin{aligned}
& \quad \zeta_{x x}^{2}=\eta_{x x}+\left[2 \eta_{x u}-\xi_{x x}^{2}\right] u_{x}-\xi^{1}{ }_{x x} u_{t}+ \\
& {\left[\eta_{u}-2 \xi^{2}\right] u_{x x}-2 \xi^{1}{ }_{x} u_{t u}+\left[\eta_{u u}-\right.} \\
& \left.2 \xi^{2}{ }_{x u}\right]\left(u_{x}\right)^{2}-2 \xi^{1}{ }_{x u} u_{t} u_{x}-\xi^{1}{ }_{u u}\left(u_{t}\right)^{3}- \\
& \xi^{2}{ }_{u u}\left(u_{t}\right)^{2} u_{x}-3 \xi^{2}{ }_{u} u_{x} u_{x x}-\xi^{1}{ }_{u u} u_{t}\left(u_{x}\right)^{2}- \\
& -\xi^{1}{ }_{u} u_{t} u_{x x}-2 \xi^{1}{ }_{u} u_{x} u_{t x} .
\end{aligned}
$$

The application of the generator to (2) leads to

$$
\eta_{x x}+\left[2 \eta_{x u}-\xi_{x x}^{2}\right] u_{x}-\xi_{x x}^{1} u_{t}+
$$

$\left[\eta_{u}-2 \xi^{2}{ }_{u}\right] u_{x x}-2 \xi^{1}{ }_{x} u_{t u}+\left[\eta_{u u}-\right.$

$\left.2 \xi^{2}{ }_{x u}\right]\left(u_{x}\right)^{2}-2 \xi^{1}{ }_{x u} u_{t} u_{x}-\xi^{1}{ }_{u u}\left(u_{t}\right)^{3}-$

$\xi_{u u}^{2}\left(u_{t}\right)^{2} u_{x}-3 \xi^{2} u_{u} u_{t}-\xi^{1}{ }_{u u} u_{t}\left(u_{x}\right)^{2}-$

$-\xi^{1}{ }_{u} u_{t} u_{x x}-2 \xi^{1}{ }_{u} u_{x} u_{-} t x-\eta_{t}-\left[\eta_{u}-\xi^{1}{ }_{t}\right] u_{t}+$

$\xi^{2}{ }_{t} u_{x}+\xi^{1}{ }_{u}\left(u_{t}\right)^{2}+\xi^{2} u_{t} u_{x}=0$,

called the determining equation. It, in turn, leads to

$$
\begin{aligned}
u_{t x} & : \xi^{1}{ }_{x}=0, \\
u_{t} & : \xi^{1}{ }_{t}-\xi^{1}{ }_{x x}-2 \xi^{2}{ }_{x}=0, \\
u_{x} & : 2 f_{x}-\xi^{2}{ }_{x x}+\xi^{2}{ }_{t}=0, \\
u & : f_{t}-f_{x x}=0, \\
1 & : g_{t}-g_{x x}=0,
\end{aligned}
$$

called monomials. Here $\eta=f u+g$. The general infinitesimals are then

$$
\begin{aligned}
& \xi^{1}=A_{1} \frac{t^{2}}{2}+A_{2} \mathrm{t}+A_{3}, \\
& \xi^{2}=\left(A_{1} t+A_{2}\right) \frac{x}{2}+B_{1} t+B_{2}
\end{aligned}
$$

and

$$
\eta=\left[-A_{1} \frac{x^{2}}{8}-B_{1} \frac{x^{2}}{2}-A_{1} \frac{t^{2}}{8}+C_{1}\right] u+g .
$$

That is,

$$
\begin{gathered}
X=\left(A_{1} \frac{t^{2}}{2}+A_{2} t+A_{3}\right) \frac{\partial}{\partial t}+ \\
\left(\left(A_{1} t+A_{2}\right) \frac{x}{2}+B_{1} t+B_{2}\right) \frac{\partial}{\partial x} \\
\left(\left[-A_{1} \frac{x^{2}}{8}-B_{1} \frac{x^{2}}{2}-A_{1} \frac{t^{2}}{8}+C_{1}\right] u+g\right) \frac{\partial}{\partial u},
\end{gathered}
$$

the general symmetry, from which the solution for (2) follows, presented in (5). 


\section{Modified Lie symmetry analysis}

Modified Lie symmetry analysis is on point transformations of point transformations. They are possible through two approaches. The first approach is direct, as it makes use of the determining equations. The second approach depends on the availability of the pure Lie symmetries, in particular, their monomials. To begin, we again start with a definition.

Definition 2. Suppose $\left(x^{1} ; \cdots ; x^{N}\right)$ is a point in $\mathrm{IR}^{N}$, and is related to another $\left(\bar{x}^{1} ; \cdots ; \bar{x}^{N}\right)$ in $\overline{\mathrm{IR}}^{N}$ by

$$
\bar{x}^{i}=f\left(x^{1} ; \cdots ; x^{N} ; a\right), i=1, \cdots, N,
$$

an invertible transformations, such that $a$ is infinitely small. Then, the symmetries that arise from the transformations

$$
\tilde{x}^{i}=f\left(\bar{x}^{1} ; \cdots ; \bar{x}^{N} ; \omega\right), i=1, \cdots, N,
$$

with the infinitesimal parameter $\omega$, are called modified Lie symmetries, or simply modified symmetries, where $\left(\tilde{x}^{1} ; \cdots ; \tilde{x}^{N}\right)$, is a point in $\widetilde{\mathrm{IR}}^{N}$.

The Lie type equations assume the form

$$
\frac{d \tilde{x}^{i}}{d \omega}=\xi^{j}\left(\bar{x}^{1} ; \cdots ; \bar{x}^{N} ; \omega\right), i, j=1, \cdots, N,
$$

with the initial conditions

$$
\left.\frac{d \tilde{x}^{i}}{d \omega}\right|_{\omega=0}=\bar{x}^{1}, i=1, \cdots, N,
$$

These then lead to the following theorem.

Theorem 1. The infinitesimals $\xi^{j}\left(\bar{x}^{1} ; \cdots ; \bar{x}^{N} ; \omega\right)$ satisfy

$$
\frac{\xi_{\omega \omega}^{j}}{\xi^{j}}=\frac{\xi_{\omega \omega \omega}^{j}}{\xi^{j} \omega}, i=1, \cdots, N .
$$

The proof follows from the Taylor-Maclaurin theorem. It acts as a lemma to the next theorem:

Theorem 2. The infinitesimals $\xi^{j}\left(\bar{x}^{1} ; \cdots ; \bar{x}^{N} ; \omega\right)$ satisfy

$$
\frac{\xi_{\bar{x}}^{j} \bar{x}^{s}}{\xi^{j}}=\frac{\xi_{\bar{x}^{s}}^{j} \bar{x}^{s} \bar{x}^{s}}{\xi_{\bar{x}}^{j}}, i, s=1, \cdots, N,
$$

where $\bar{x}^{s}$ is a variable.

The proof also follows from the TaylorMaclaurin theorem. This author discusses the solutions of (29) and (30) extensively in [8] and [9]. These we solve after addressing some errors in Leonhard Euler's works. Other authors did also encounter other errors in this great scholar's works. These include Elgindi et. al., see [10] and [11]. The solution has the form

$$
\xi^{j}=a \frac{\sin (i \omega[x+\phi])}{i \omega},
$$

where $a, \phi$ and $\omega$ are integration constants. It is what we use to get the modified symmetries easily. The next property to use is that when all transformation are zero, all symmetries are equal, as explained in the next theorem.

Theorem 3. Suppose $\xi^{j}\left(x^{1} ; \cdots ; x^{N}\right)$ is an infinitely differentiable infinitesimal from the Cartesian system $\operatorname{IR}^{N}$ to $\overline{\mathrm{IR}}^{N}$, and $\tilde{\xi}^{j}\left(\bar{x}^{1} ; \cdots ; \bar{x}^{N} ; \omega\right)$ also an infinitely differentiable infinitesimal from $\overline{\mathrm{IR}}^{N}$ to $\widetilde{\mathrm{IR}}^{N}$. Then the following is true.

$$
\left.\frac{\partial^{k} \tilde{x}^{j}}{\partial\left(\bar{x}^{s}\right)^{k}}\right|_{\omega=0}=\frac{\partial^{k} \xi^{j}}{d\left(x^{s}\right)^{k}}, \quad s=1, \cdots, N,
$$

for all $k$.

\subsection{The direct approach}

This approach requires that we first go through all the monomials (16), (17), (18), (19) and (20), and determine where the solution (31) applies. A quick review reveals it applies at monomial (16), in that case the monomial can be differentiated to give

$$
\xi^{1}{ }_{x x}=0 .
$$

With the introduction of an infinitesimal parameter $\omega$, it becomes

$$
\tilde{\xi}_{x x}^{1}=-\omega^{2} \tilde{\xi}^{1} .
$$

Note that $\xi^{1}$ now assumes the form $\tilde{\xi}^{1}$ to indicate the presence of the infinitesimal parameter of modified symmetries, and that the resulting symmetries will be modified symmetries.

This then transforms the determining equations (15) into

$$
\begin{aligned}
& \tilde{\eta}_{x x}+\left[\begin{array}{ll}
2 \tilde{\eta}_{x u} & -\tilde{\xi}_{x x}^{2}
\end{array}\right] u_{x}+\omega^{2} \tilde{\xi}^{1} u_{t}+ \\
& {\left[\eta_{u}-2 \tilde{\xi}_{u}^{2}\right] u_{x x}-2 \tilde{\xi}^{1}{ }_{x} u_{t u}+\left[\tilde{\eta}_{u u}-\right.} \\
& \left.2 \tilde{\xi}_{x u}^{2}\right]\left(u_{x}\right)^{2}-2 \tilde{\xi}_{x u}^{1} u_{t} u_{x}-\tilde{\xi}^{1}{ }_{u u}\left(u_{t}\right)^{3}- \\
& \tilde{\xi}^{2}{ }_{u u}\left(u_{t}\right)^{2} u_{x}-3 \tilde{\xi}^{2}{ }_{u} u_{x} u_{t}-\tilde{\xi}^{1}{ }_{u u} u_{t}\left(u_{x}\right)^{2}- \\
& -\tilde{\xi}^{1} u_{u} u_{t} u_{x x}-2 \tilde{\xi}^{1}{ }_{u} u_{x} u_{t x-\eta_{t}}-\left[\tilde{\eta}_{u}-\right. \\
& \left.\tilde{\xi}^{1}{ }_{t}\right] u_{t}+\tilde{\xi}^{2}{ }_{t} u_{x}+\tilde{\xi}^{1}{ }_{u}\left(u_{t}\right)^{2}+\tilde{\xi}^{2}{ }_{u} u_{t} u_{x}=0 \text {. }
\end{aligned}
$$

\subsubsection{Multi-parameter symmetries}

A closer look at the second monomial (17) reveals that the first, (16), reduces it into

$$
\xi^{2}{ }_{x x}=0 \text {, }
$$

so that, with the introduction of a second infinitesimal parameter $\omega_{1}$, it becomes

$$
\tilde{\xi}_{x x}^{2}=-\omega_{1}^{2} \tilde{\xi}^{2} .
$$

If we rewrite (34) here with $\omega_{2}$, we then have

$$
\tilde{\xi}_{x x}^{1}=-\omega_{2}^{2} \tilde{\xi}^{1},
$$


so that from the system, (36) and (37), is a multiparameter system, from which modified symmetries follow, and follow from the determining equation

$$
\begin{aligned}
& \tilde{\eta}_{x x}+\left[2 \tilde{\eta}_{x u}+\omega_{1}^{2} \tilde{\xi}^{2}\right] u_{x}+\omega_{2}^{2} \tilde{\xi}^{1} u_{t}+ \\
& {\left[\eta_{u}-2 \tilde{\xi}^{2}{ }_{u}\right] u_{x x}-2 \tilde{\xi}^{1}{ }_{x} u_{t u}+\left[\tilde{\eta}_{u u}-\right.} \\
& \left.2 \tilde{\xi}^{2}{ }_{x u}\right]\left(u_{x}\right)^{2}-2 \tilde{\xi}^{1}{ }_{x u} u_{t} u_{x}-\tilde{\xi}^{1}{ }_{u u}\left(u_{t}\right)^{3}- \\
& \tilde{\xi}^{2}{ }_{u u}\left(u_{t}\right)^{2} u_{x}-3 \tilde{\xi}^{2}{ }_{u} u_{x} u_{t}-\tilde{\xi}^{1}{ }_{u u} u_{t}\left(u_{x}\right)^{2}- \\
& -\tilde{\xi}^{1}{ }_{u} u_{t} u_{x x}-2 \tilde{\xi}^{1}{ }_{u} u_{x} u_{t x-\eta_{t}}-\left[\tilde{\eta}_{u}-\right. \\
& \left.\tilde{\xi}^{1}{ }_{t}\right] u_{t}+\tilde{\xi}^{2}{ }_{t} u_{x}+\tilde{\xi}^{1}{ }_{u}\left(u_{t}\right)^{2}+\tilde{\xi}^{2}{ }_{u} u_{t} u_{x}=0 .
\end{aligned}
$$

This is another way of determining the modified symmetries.

\subsection{The algebraic approach}

Theorem 3 leads to the modified symmetry

$\tilde{X}=\tilde{\xi}^{1} \frac{\partial}{\partial t}+\tilde{\xi}^{2} \frac{\partial}{\partial x}+\tilde{\eta} \frac{\partial}{\partial u}$,

with the infinitesimals given as

$$
\begin{aligned}
& \tilde{\xi}^{1}=-2 \phi\left(\frac{C_{0}}{\omega^{4}\left(\omega^{2}+1\right)}\right) e^{\omega^{2} t} \sin \left(\frac{\omega x}{i}\right)- \\
& 2 \phi\left(C_{1} t-C_{2} e^{-t}\right) \sin \left(\frac{\omega x}{i}\right)+ \\
& 2\left(\frac{D_{0}}{\omega^{4}\left(\omega^{2}-1\right)}\right) e^{\omega^{2} t} \cos \left(\frac{\omega x}{i}\right)+2\left(D_{1} t+\right. \\
& \left.D_{2} e^{t}\right) \cos \left(\frac{\omega x}{i}\right)+A_{0}, \\
& \tilde{\xi}^{2}=\frac{i \phi}{\omega}\left(\frac{C_{0}}{\omega^{2}\left(\omega^{2}+1\right)}\right) e^{\omega^{2} t} \cos \left(\frac{\omega x}{i}\right)+\frac{i \phi}{\omega}\left(\mathrm{C}_{1} \mathrm{t}+\right. \\
& \left.\mathrm{C}_{2} \mathrm{e}^{-\mathrm{t}}\right) \cos \left(\frac{\omega \mathrm{x}}{\mathrm{i}}\right)+\frac{i}{\omega}\left(\frac{\mathrm{D}_{0}}{\omega^{2}\left(\omega^{2}-1\right)}\right) \sin \left(\frac{\omega x}{\mathrm{i}}\right)+ \\
& \frac{i}{\omega}\left(D_{1} t+D_{2} e^{t}\right) \sin \left(\frac{\omega x}{i}\right)+A_{0},
\end{aligned}
$$

and

$$
\begin{array}{r}
\tilde{\eta}=-\left(\phi\left(\frac{C_{0}}{2}\right) e^{\omega^{2} t} \sin \left(\frac{\omega x}{i}\right)+\right. \\
\left.\phi\left(\frac{D_{0}}{2}\right) e^{\omega^{2} t} \cos \left(\frac{\omega x}{i}\right)-\frac{B_{0}}{2}\right) u+g .
\end{array}
$$

\section{Group invariant solutions}

We determine solutions using two procedures. That is, after having determined the invariants. The first procedure is through mean value theorems, and the second through differentiable topological manifolds. A closer look at the infinitesimals $\tilde{\xi}^{1}$ and $\tilde{\xi}^{2}$ reveals that

$$
\tilde{\xi}^{1}=\frac{\partial \tilde{\xi}^{2}}{\partial x}
$$

This makes it easier to determine invariants that encompasses all parameters.
From the generator (40), we get the system

$$
\frac{d t}{\widetilde{\xi}^{1}}=\frac{d x}{\widetilde{\xi}^{2}}=\frac{d u}{\widetilde{\eta}} .
$$

Alternatively

$$
\frac{d t}{\widetilde{\xi}^{1}}=\frac{d x}{\widetilde{\xi}^{2}}=\frac{d u}{f u+g^{\prime}}
$$

so that the first invariant $\chi$ is given by

$$
\begin{aligned}
& \quad \chi=\ln \left(\frac{i \phi}{\omega}\left(\frac{C_{0}}{\omega^{2}\left(\omega^{2}+1\right)}\right) e^{\omega^{2} t} \cos \left(\frac{\omega x}{i}\right)+\right. \\
& \frac{i \phi}{\omega}\left(\mathrm{C}_{1} \mathrm{t}+\mathrm{C}_{2} \mathrm{e}^{-\mathrm{t}}\right) \cos \left(\frac{\omega \mathrm{x}}{\mathrm{i}}\right)+ \\
& \frac{i}{\omega}\left(\frac{\mathrm{D}_{0}}{\omega^{2}\left(\omega^{2}-1\right)}\right) \sin \left(\frac{\omega \mathrm{x}}{\mathrm{i}}\right)+\frac{i}{\omega}\left(D_{1} t+\right. \\
& \left.\left.D_{2} e^{t}\right) \sin \left(\frac{\omega x}{i}\right)+A_{0}\right),
\end{aligned}
$$

and the second $\psi=\psi(\chi)$ by

$$
\frac{\tilde{\xi}^{1}}{f} \ln \left(u+\frac{g}{f}\right)=t+\psi,
$$

or

$$
u=\exp \left[(t+\psi) \frac{f}{\tilde{\xi}^{1}}\right]-\frac{g}{f} .
$$

This then leads to

$$
\begin{gathered}
u_{t}=\exp \left[(t+\psi) \frac{f}{\tilde{\xi}^{1}}\right] \frac{\partial}{\partial t}\left[(t+\psi) \frac{f}{\tilde{\xi}^{1}}\right]-\frac{\partial}{\partial t}\left[\frac{g}{f}\right] \\
u_{x}=\exp \left[(t+\psi(1)) \frac{f}{\tilde{\xi}^{1}}\right] \frac{\partial}{\partial x}\left[(t+\psi) \frac{f}{\tilde{\xi}^{1}}\right]-\frac{\partial}{\partial x}\left[\frac{g}{f}\right]
\end{gathered}
$$
and

$$
\begin{aligned}
& u_{x x}=\exp \llbracket\left[(t+\psi) \frac{f}{\tilde{\xi}^{1}}\right]\left[\frac{\partial}{\partial x} \rrbracket\left[\psi \frac{f}{\tilde{\xi}^{1}}\right]\right]^{2}+ \\
& \exp \left[(t+\psi) \frac{f}{\tilde{\xi}^{1}}\right] \frac{\partial^{2}}{\partial x^{2}}\left[\psi \frac{f}{\tilde{\xi}^{1}}\right]+-\frac{\partial^{2}}{\partial x^{2}}\left[\frac{g}{f}\right] .
\end{aligned}
$$

The linear heat equation now assumes the form

$$
\begin{aligned}
& \exp \left[(t+\psi) \frac{f}{\tilde{\xi}^{1}}\right] \frac{\partial}{\partial t}\left[(t+\psi) \frac{f}{\tilde{\xi}^{1}}\right]-\frac{\partial}{\partial t}\left[\frac{g}{f}\right]= \\
& \exp \llbracket\left[(t+\psi) \frac{f}{\tilde{\xi}^{1}}\right]\left[\frac{\partial}{\partial x}\right)\left[\left[\psi \frac{f}{\tilde{\xi}^{1}}\right]\right]^{2}+\exp [(t+ \\
& \left.\psi) \frac{f}{\tilde{\xi}^{1}}\right] \frac{\partial^{2}}{\partial x^{2}}\left[\psi \frac{f}{\tilde{\xi}^{1}}\right]+-\frac{\partial^{2}}{\partial x^{2}}\left[\frac{g}{f}\right] .
\end{aligned}
$$

\subsection{The case $C_{0} \neq 0$}

The case where $C_{0} \neq 0$ and all other parameters being zero, reduces (53) to

$$
\begin{aligned}
& \left(\omega^{2}\left[e^{\omega^{2} t}-\chi^{2}\right]\right) \frac{\mathrm{d}^{2} \psi}{d \chi^{2}}+\left(\frac{\omega^{2}}{2} \chi\right) \frac{d \psi}{d \chi}+ \\
& \left(\frac{\omega^{2}\left[\omega^{4}-1\right]}{2}\right) \psi=0,
\end{aligned}
$$


with

$$
u=\psi e^{\omega^{4}\left(\omega^{2}-1\right) t / 4}
$$

and

$$
\chi=e^{\omega^{2} t / 2} \sin \left(\frac{\omega x}{i}\right)
$$

\subsubsection{The mean value theorems' approach}

We note that (54) simplifies easily through limit principles: The limit of a sum is equal to the sum of limits, and so is a limit of a product equal to a product of limits. Isolating the second term and applying these principles then leads to

$$
\left(\frac{\omega^{2}}{2} \chi\right) \frac{d \psi}{d \chi}=\left(\frac{0}{2} \chi\right) \frac{d \psi}{d \chi}=0 .
$$

This reduces (54) to

$$
\left(\left[\omega^{2} e^{\omega^{2} t}-\omega^{2} \chi^{2}\right]\right) \frac{\mathrm{d}^{2} \psi}{d \chi^{2}}+\left(\frac{\omega^{2}\left[\omega^{4}-1\right]}{2}\right) \psi=0 .
$$

It can be re-written in the form

$$
\omega^{2} e^{\omega^{2} t} \frac{\mathrm{d}^{2} \psi}{d \chi^{2}}+\left(\frac{\omega^{2}\left[\omega^{4}-1\right]}{2}\right) \psi=0 .
$$

Applying the formula we developed in [11] and [12], we have

$\psi=\frac{e^{r \chi}}{\omega}$.

This leads to

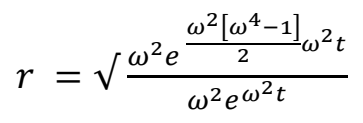

or

$r=\sqrt{\frac{\omega^{2}\left(\operatorname{Cos}\left[\frac{\omega^{2}\left[\omega^{4}-1\right]}{2 i} \omega^{2} t\right]+i \operatorname{Sin}\left[\frac{\omega^{2}\left[\omega^{4}-1\right]}{2 i} \omega^{2} t\right]\right)}{\omega^{2}\left(e^{\omega^{2} t}\right) \omega^{2}\left(\operatorname{Cos}\left[\omega^{2}\left(e^{\omega^{2} t}\right) / i\right]+i \operatorname{Sin}\left[\omega^{2}\left(e^{\omega^{2} t}\right) / i\right]\right)}}$

After a very long analysis, we get

$$
u=G_{0} \frac{x}{2 \sqrt{t}} e^{-x^{2} /(4 t)},
$$

where $G_{0}$ is the integration constant.

\subsubsection{The approach through manifolds}

To use differentiable topological manifolds to solve (54), we first have to make a connection between the elementary mathematical level we are at and equivalent classes, found deep inside differentiable topological manifolds. This author addressed this extensively in [12] and [13]. Here we simply apply what is developed there to (54).

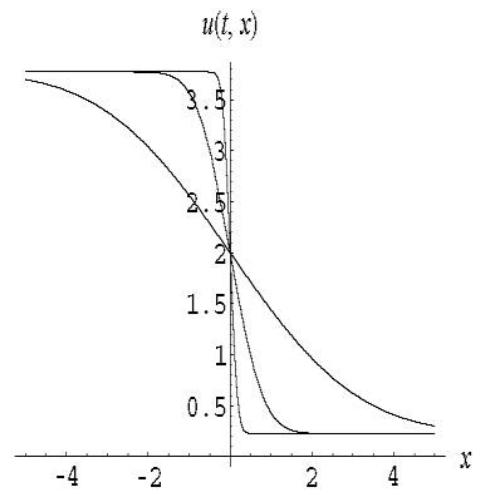

Fig. 1. A plot of the solution we obtained for (2), presented in (58).

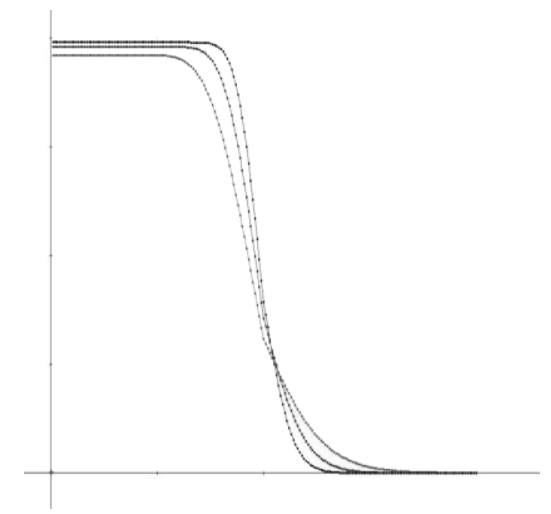

Fig. 2. A plot of the solution obtained for (2) by Fassari and Rinaldi [14]. They obtained using numerical techniques. Analytical are preferable because their a guide on what next steps to follow.

136




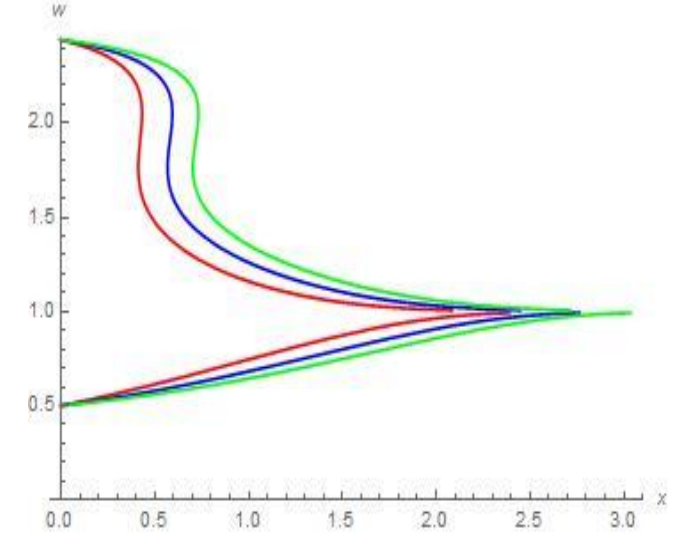

Fig. 3. A plot of the solution for (1), obtained under the transformation in (3), of (56).n
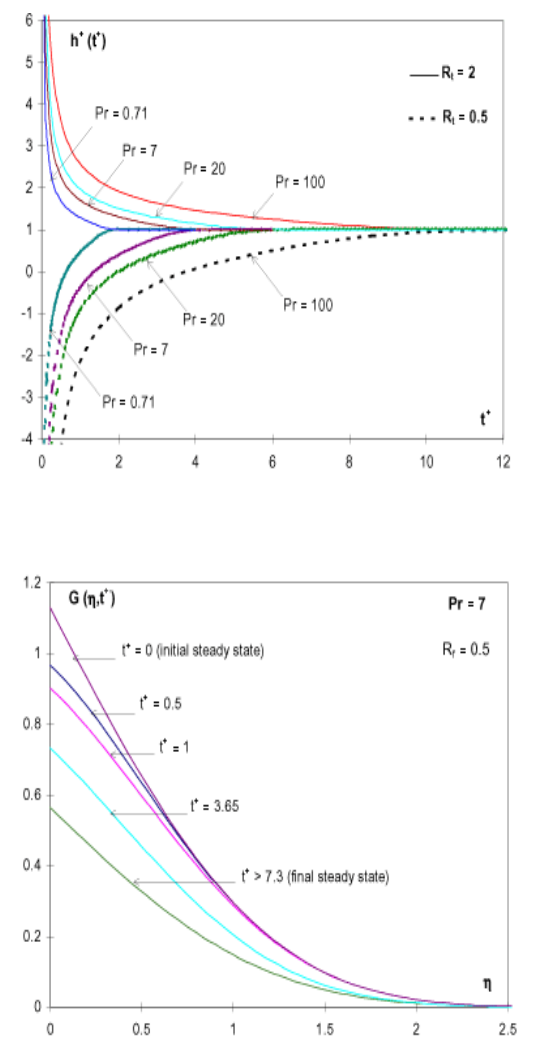

Figure 2.9. Transient temperature profiles for a negative step change in the plate heat flux.

Fig. 4. A plot of the solution [1](Their Figure 2.9), matching our Figure 3, especially the presence of the singularity on the vertical axes.
The application of equivalence classes from [12] and [13] to (54)

We seek equivalent classes as solutions of (54). One possible case, is from the equation

$$
\frac{[\psi]^{(3)}}{[\psi]^{\prime}}=\frac{[\psi]^{(4)}}{[\psi]^{\prime \prime}},
$$

where $[\psi]=[\psi]([\chi])$, whose solution is

$$
[\psi]^{\prime}=[G] \frac{\sin (i[\lambda]([\chi]+[\phi]))}{i[\lambda]},
$$

Again from [8] and [9], with

$$
i[\lambda]([\chi]+[\phi])=K \pi,
$$

for all integer values of $\mathrm{K}$. That is,

$$
[\psi]=[G] \frac{\sin (i[\lambda]([\chi]+[\phi]))}{i[\lambda]}+[H] .
$$

Substituting these into (54) yields

$$
\left(\omega^{2}\left[e^{\omega^{2} t}-\chi^{2}\right]\right)[G]+\left(\frac{\omega^{2}\left[\omega^{4}-1\right]}{2}\right)\left(\frac{[G]}{i[\lambda]}+[H]\right)=
$$

0 .

Substituting these into (54) yields

$$
[H]=-2 \frac{\left(\omega^{2}\left[e^{\omega^{2} t}-\chi^{2}\right]\right)[G]}{\left[\omega^{2}\left[\omega^{4}-1\right]\right]}-\frac{[G]}{i[\lambda]}
$$

Hence,

$$
[H]=-2 \frac{\left[\left(\omega^{2}\left[e^{\omega^{2} t}-\left[\chi^{2}\right]\right]\right)\right][G]}{\left[\omega^{2}\left[\omega^{4}-1\right]\right]}-\frac{[G]}{i[\omega]^{\prime}}
$$

or

$$
[H]=-2 \frac{\left[\left(\omega^{2}\left[e^{\omega^{2} t}-(K \pi)^{2}\right]\right)\right][G]}{\left[\omega^{2}\left[\omega^{4}-1\right]\right]}-\frac{[G]}{i[\omega]}
$$

That is,

$$
\begin{aligned}
& {[\psi]=[G] \frac{\sin (i[\omega]([\chi]+[\phi]))}{i[\omega]}+} \\
& -2 \frac{\left[\left(\omega^{2}\left[e^{\omega^{2} t}-(K \pi)^{2}\right]\right)\right][G]}{\left[\omega^{2}\left[\omega^{4}-1\right]\right]}-\frac{[G]}{i[\omega]}
\end{aligned}
$$

The next step is to integrate (54) at every point given by $[\psi]$. That is, 


$$
\begin{gathered}
\int\left(\left(\omega^{2}\left[e^{\omega^{2} t}-\chi^{2}\right]\right) \frac{\mathrm{d}^{2} \psi}{d \chi^{2}}+\left(\frac{\omega^{2}}{2} \chi\right) \frac{d \psi}{d \chi}+\right. \\
\left.\left(\frac{\omega^{2}\left[\omega^{4}-1\right]}{2}\right) \psi\right) d \chi+J_{1}=0
\end{gathered}
$$

and

$$
\begin{gathered}
\int\left(\int \left(\left(\omega^{2}\left[e^{\omega^{2} t}-\chi^{2}\right]\right) \frac{\mathrm{d}^{2} \psi}{d \chi^{2}}+\left(\frac{\omega^{2}}{2} \chi\right) \frac{d \psi}{d \chi}+\right.\right. \\
\left.\left.\left(\frac{\omega^{2}\left[\omega^{4}-1\right]}{2}\right) \psi\right) d \chi\right) d \chi+J_{1} \chi+J_{2}=0
\end{gathered}
$$

where $J_{1}$ and $J_{2}$ are integration constants. Hence,

$$
\begin{gathered}
\psi=-\int\left\{\int \left\{\left(\frac{1}{2\left[e^{\omega^{2} t}-\chi^{2}\right]} \chi\right) \frac{d \psi}{d \chi}+\right.\right. \\
\left.\left.\left(\frac{\omega^{4}-1}{2\left[e^{\omega^{2} t}-\chi^{2}\right]}\right) \psi\right\} d \chi\right\} d \chi+J_{1} \chi+J_{2} .
\end{gathered}
$$

$\psi=\left(D_{1} \frac{\left(\frac{T}{\beta}\right)}{X}+D_{2}\right) e^{-\left(\frac{T}{\beta}\right)^{2} /(4 X)}$

From the plot of $\psi \psi^{*}$ against $X$, while keeping $T=$ 1 , has two peaks, implying the two positions. It gives

$d_{0}=\lambda \beta$.

The quantity $\psi^{*}$ is the complex conjugate of $\psi$. And $d_{0}$ is the separation distance in the quantum superposition. For a beta particle moving at the speed of light, with frequency $f=10^{18} \mathrm{~Hz}$, we get $d_{0}=4 \mathrm{~nm}$. All these values fall within nanoscience ranges.

this contribution, we demonstrated the edge our modified symmetries has over the regular Lie symmetries. This we did by solving the heat equation (2), and nonlinear equation (1).

\section{Applications}

As was briefly discussed in the Introduction, the heat equation has a number of applications. We will here apply it to quantum mechanics.

\subsection{The quantum superposition principle}

The quantum superposition principle, states that a particle can occupy two different positions at once. It is never presented in mathematical terms. We will here develop a formula for it.

Note that the Schrodinger equation for a free particle, has the form

$-\frac{h}{2 \pi m} \frac{\partial^{2} \psi}{\partial \chi^{2}}=i \frac{\partial \psi}{\partial t}$.

Where $h$ is Planck's constant, $m$ particle mass $t$ time, $\chi$ the spatial variable, and $\psi$ the wave function. Introducing dimensionless variables $T=$ $\frac{t}{T}=f t$ and $X=\chi / \lambda$. This transforms (71) into

$\frac{\partial^{2} \psi}{\partial X^{2}}=\beta \frac{\partial \psi}{\partial t}$

with

$\beta=i \frac{4 \pi m}{h} \frac{T^{2}}{\lambda}$.

The solution through symmetry $X_{1}$ is

\section{Discussion and Conclusion}

In this contribution, we demonstrated the edge our modified symmetries has over the regular Lie symmetries. This we did by solving the heat equation (2), and nonlinear equation (1).

In application, the heat equation finds uses in financial engineering, or referred often as financial mathematics.

We revealed that there hidden solutions, Thus we conclude that they can be useful in better explaining options and derivatives.

We also determined the separation distance in the quantum superposition principle.

\section{References:}

[1] Lie S., On integration of a class of linear partial differential equations by means of definite integrals, Arch. Math., Vol.3, 1881, pp. 328368.

[2] Ovsiannikov, L.V., Group properties of nonlinear heat equation, Dokl. AN SSSR, VOL.125, NO.3, 1959, pp. 492-495.

[3] Amlan K.H., Andronikos Paliathanasis, P. and Leach, P.G.L., Similarity solutions and conservation laws for the Beam Equations: a complete study, math-ph, 2020, arXiv. 
[4] Aeeman F., Mahomed, F.M., Khalique, C.M., Conditional symmetries of nonlinear thirdorder ordinary differential equations, Discrete $\&$ Continuous Dynamical Systems - S, VOL.11, 2018, pp. 655

[5] Wafo Soh, C., Automatic classification of automorphisms of lower-dimensional Lie algebras, Experimental Mathematics, 2017, DOI: $10.1080 / 10586458.2018 .1516582$.

[6] Anco, S. and Kara, A. Symmetry-invariant conservation laws of partial differential equations, European Journal of Applied Mathematics. VOL. 1, NO. 29, 2020, pp. 78117.

[7] M.A. Latif, M.A., Dragomir, S.S, and Momoniat, E. Some q-analogues of HermiteHadamard inequality of functions of two variables on finite rectangles in the plane, Journal of King Saud University - Science, VOL. 29, NO. 29, 2017, pp. 263-273.

[8] Gainetdinova, A. and Gazizov, R., Integrating of systems of two second-order ordinary differential equations with a small parameter that admit four essential operators, Sibirskie Elektronnye Matematicheskie Izvestiya, NO. 17, 2020, pp. 604-614, DOI: 10.33048/semi.2020.17.039.
[9] Amlan K.H., Andronikos Paliathanasis, P. and Leach, P.G.L., Similarity solutions and conservation laws for the Beam Equations: a complete study, math-ph, 2020, arXiv.

[10] Edelstein, RM, Govinder, KS. On the method of preliminary group classification applied to the nonlinear heat equation $\mathrm{ut}=\mathrm{f}(\mathrm{x}, \mathrm{ux}) \mathrm{ux}$ $\mathrm{x}+\mathrm{g}(\mathrm{x}, \mathrm{u}$ x $)$. Math Meth Appl Sci., 2020, 43: $5927 ?$ ? 5940. https://doi.org/10.1002/mma.6333

[11] Manale, J.M., On Errors in Euler's Formula for Solving ODEs, International Journal of Mathematical and Computational Methods, VOL.5, 2020, pp.1-3.

[12] Manale, J.M., (2020). On errors in Euler's complex exponent and formula for solving ODEs, Journal of Physics: Conference Series, 2020, DOI: 1564. 012021. 10.1088/1742$6596 / 1564 / 1 / 012021$.

\section{Creative Commons Attribution License 4.0 (Attribution 4.0 International, CC BY 4.0)}

This article is published under the terms of the Creative Commons Attribution License 4.0

https://creativecommons.org/licenses/by/4.0/deed.en_US 